\title{
Electrochemical Flow Cell to Detect Li-ion Battery Crosstalk Reactions
}

\author{
Oliver C. Harris, Maureen H. Tang* \\ Department of Chemical \&3 Biological Engineering \\ Drexel University, Philadelphia, PA 19104
}

August 12, 2020

\begin{abstract}
While the Li-ion battery has been engineered over the last four decades to improve energy capacity, power density, and device safety, the useful lifetime of this essential energy storage technology has not progressed as much. This is largely due to experimental challenges of studying, characterizing, and understanding the SEI: the battery 'component' most vital to ageing and failure. More importantly for the goal of improving Li-ion battery lifetime, researchers have lacked adequate diagnostic tools for studying how the SEI fails. Here we demonstrate a prototype electrochemical flow cell for the specific application of detecting crosstalk reactions in advanced Li-ion battery chemistries. We develop a generator-collector approach to understanding battery crosstalk and leverage finite-element simulations to guide design of this novel reactor. After calibrating the device using a known redox couple, the device is cycled under varying electrode configurations to detect capacity fade induced by the metal dissolution crosstalk mechanism. The path forward will involve adding new product detection capabilities and engineering a reactor environment that replicates a sealed Li-ion battery.
\end{abstract}

*Email: mhtang@drexel.edu 


\section{Introduction}

Since the Li-ion battery (LIB) was first commercialized in 1981, almost every design aspect has been optimized to increase the power and energy densities, operating voltage, and safety of these ubiquitous energy storage devices. One area remains a significant challenge for battery researchers to optimize: battery lifetime. This is because LIB lifetime is not something easily designed for a priori, since lifetime is a direct result of side reactions. Side reactions are inherently undesirable because they consume charge without contributing to the energy storage capacity of the battery, thus they decrease the coulombic efficiency of the battery.

Coulombic efficiency (CE) has long been a catch-all metric for describing battery performance, and is routinely used as an initial estimate of a battery's state of health and likelihood of failure. However, recent evidence has emerged that casts doubt on the utility of this singular metric, specifically that certain side reactions in a battery may lower the coulombic efficiency without causing failure or negatively impacting battery lifetime.[1] These certain side reactions can be classified as crosstalk reactions since they often involve products formed at one electrode reacting at the opposite electrode.

The most common crosstalk reactions in Li-ion batteries are detrimental to performance and lifetime, however some crosstalk reactions are not wholly pernicious and may even provide a net benefit to battery lifetime. For instance, $\mathrm{CO}_{2}$ is suspected of playing an important role in battery lifetime, even though losing electrons to $\mathrm{CO}_{2}$ reactions may initially appear detrimental, specifically in coulombic efficiency analysis. Recent work has demonstrated that $\mathrm{CO}_{2}$ in LIBs can help passivate electrode surfaces by promoting $\mathrm{Li}_{2} \mathrm{CO}_{3}$ formation. Additionally, there may be evidence that $\mathrm{CO}_{2}$ scavenges protons, which would clearly have benefits for delaying capacity fade. A crosstalk reaction that does not cause capacity fade manifests in a lower observed coulombic efficiency but can be considered benign or at best neutral to battery performance and lifetime. However, most observed crosstalk reactions are, in fact, firmly detrimental to battery performance and lifetime, and require devoted study and consideration.

One major roadblock to understanding this class of side reactions is the battery itself. While an incredible device for energy storage, a two-electrode battery is extremely limiting as an analytical tool for understanding reaction kinetics and thermodynamics. With only two electrodes and no reference thermodynamic potential, deconvoluting reaction networks that involve participants originating from the other electrode is untenable. Additionally, practical batteries are poorly-mixed batch reactors, which makes reaction 
product determination and kinetic analysis nearly impossible. Researchers in the electrocatalysis field have been successful in designing flow reactors to study complex reaction pathways that would be unfeasible under stagnant, batch conditions. In order to start to understand complex crosstalk mechanisms and begin the vital task of mapping out these reaction networks, the battery as an analytic tool must be discarded and a new platform for electroanalysis of battery reactions must be prioritized and developed.

Several non-traditional reactors already exist that have been designed and developed to study battery reactions and interfaces. For instance, interdigitated electrode arrays are typically unstirred batch reactors with two electrodes. Each electrode is patterned via lithography to create high aspectratio 'fingers' that are separated by the 'fingers' of the counter-electrode, creating a series of interdigitated electrodes. These devices are useful for studying reaction product transport, since the inter-electrode distance is small enough for rapid product diffusion. This reactor design is currently limiting in electrode material selection and may become a more powerful tool if commercial battery electrodes can be used. The dual-band microfluidic cell developed by Crooks and colleagues also suffers from limited electrode material options, but is an important example of a novel flow device for characterizing electrochemical reactions.[2] Lastly, while the rotating ring-disk electrode (RRDE) has been used to study various battery reactions, the platform also has limited electrode material variability, and more importantly has an unacceptably low surface-area to volume ratio when compared to practical batteries. [3-5] Thus, researchers are still working toward developing a novel reactor specifically designed to study and deconvolute complex reaction networks in batteries.

In this work we present the development of an electrochemical flow cell for studying crosstalk reactions in a LIB environment. The flow cell is characterized in various operation modes and a proof-of-concept series of tests are performed. We report that the flow cell can detect crosstalk reactions, specifically metal dissolution from a high-voltage positive electrode and deposition at a downstream negative electrode. We describe methods for utilizing the flow cell to perform collector-generator experiments and outline a path forward for multifunctional flow cells as a new platform for analytical electrochemistry. 


\section{Methods and materials}

\subsection{Electrode preparation}

Working electrodes were prepared by mechanically cutting electrode sheets to a desired geometry. The negative working electrode was single-layer graphite coated on $\mathrm{Cu}$ foil (MTI) and the positive electrode was NMC-532 $\left(\mathrm{Li}\left(\mathrm{Ni}_{0.5} \mathrm{Mn}_{0.3} \mathrm{Co}_{0.2}\right) \mathrm{O}_{2}\right)$ coated on $\mathrm{Al}$ foil $(\mathrm{MTI})$.

The reference and counter electrodes were semi-delithiated $\mathrm{Li}_{0.5} \mathrm{FePO}_{4}$, prepared by following a similar procedure to Wandt et al.[6] First, a portion of $\mathrm{Li}_{1} \mathrm{FePO}_{4}$ powder (MTI) was stirred for 72 hours in solution with excess potassium peroxodisulfate to chemically delithiate the powder to $\mathrm{Li}_{0} \mathrm{FePO}_{4}$. The fully-delithiated $\mathrm{Li}_{0} \mathrm{FePO}_{4}$ was filtered out of solution, washed with deionized water, and then dried under vacuum at $70{ }^{\circ} \mathrm{C}$ overnight. The dried powder was then mixed with fully-lithiated $\mathrm{Li}_{1} \mathrm{FePO}_{4}$ in an equimolar ratio, and thoroughly ball-milled to yield semi-delithiated $\mathrm{Li}_{0.5} \mathrm{FePO}_{4}$. An overhead mixer stirred $\mathrm{Li}_{0.5} \mathrm{FePO}_{4}$ powder, deionized water, polyethylene oxide, and carbon black to form a slurry that was then cast onto aluminum foil (MTI) via a doctor blade and automatic coater (TOB Energy). The electrode sheet was dried overnight under vacuum before being cut to make reference and counter electrode strips. Semi-delithiated $\mathrm{Li}_{0.5} \mathrm{FePO}_{4}$ was selected because it can be safely manipulated outside of an inert glovebox and typically exhibits a wide voltage plateau.

\subsection{Flow cell materials and fabrication}

The flow cell prototypes were constructed using glass microscope slides as the bottom and top backing plates. Starting with the bottom plate, copper and aluminum conductive tape were placed to cover the footprint area of the working and reference electrodes, followed by application of colloidal silver paste (Ted Pella) on the conductive tapes. Precut working and reference electrodes were then aligned and adhered to the tapes by bonding with the silver adhesive, yielding a bottom half cell as seen in Figure 1a.

To construct the flow channel, layers of patterned $25 \mu \mathrm{m}$ hot-melt sealing film (Meltonix 1170, Solaronix) were stacked on top of the electrodes and were tap-sealed using a soldering iron, then fully sealed by heating in a 100 ${ }^{\circ} \mathrm{C}$ oven for 40 minutes. The sealing film was lasercut prior to cell fabrication in order to create a flow field in each layer. When layers are aligned and stacked, the cut portion creates a flow channel over the electrodes from the inlet port to the outlet port. This approach allows for facile manipulation 
of channel height in $25 \mu \mathrm{m}$ increments. Assembled layers of the sealing film and the inlet and outlet tubing are shown in Figure 1b.

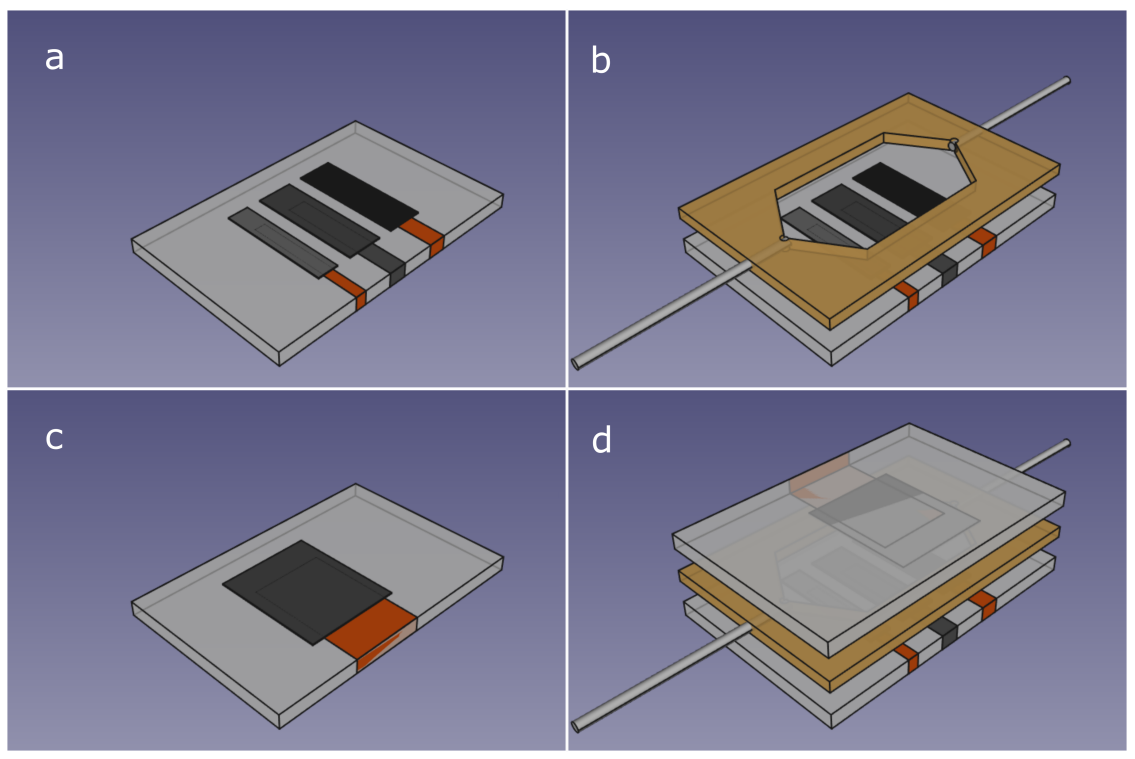

Figure 1: Schematic of disassembled flow cell parts. (a) Bottom half consisting of backing plate, conductive tapes, conductive adhesives, and working and reference electrodes; (b) Flow channel consisting of two layers of lasercut sealing film and tubing; (c) Top half consisting of backing plate, conductive tape, conductive adhesive, and a counter electrode; (d) Stacked cell parts prior to final sealing and enclosure.

The top-half of the flow cells were fabricated similarly to the bottom half (Figure 1c), but included a counter electrode. Then, the two halves of the flow cell were aligned and sealed again in a $100{ }^{\circ} \mathrm{C}$ oven for 40 minutes. After inlet and outlet fittings are affixed to the inlet and outlet ports, a secondary sealing procedure is performed whereby a commercial epoxy is applied along the perimeter of cell layers to further prevent leaking (Figure 2a). Finally, the entire assembly is submerged in liquid PDMS contained within a mold and then cured at $100^{\circ} \mathrm{C}$ for 40 minutes. This final step helps maintain mechanical durability during device handling and helps prevent leaking. Figure 1d shows a schematic representation of a full-cell and Figure $2 \mathrm{~b}$ shows a photograph of an assembled cell.

An alternative fabrication approach was explored where a 3D-printed flow channel was used in lieu of stacked sealing film as previously described. Figures $2 \mathrm{c}$ and $2 \mathrm{~d}$ show an assembled flow cell using this new fabrication 


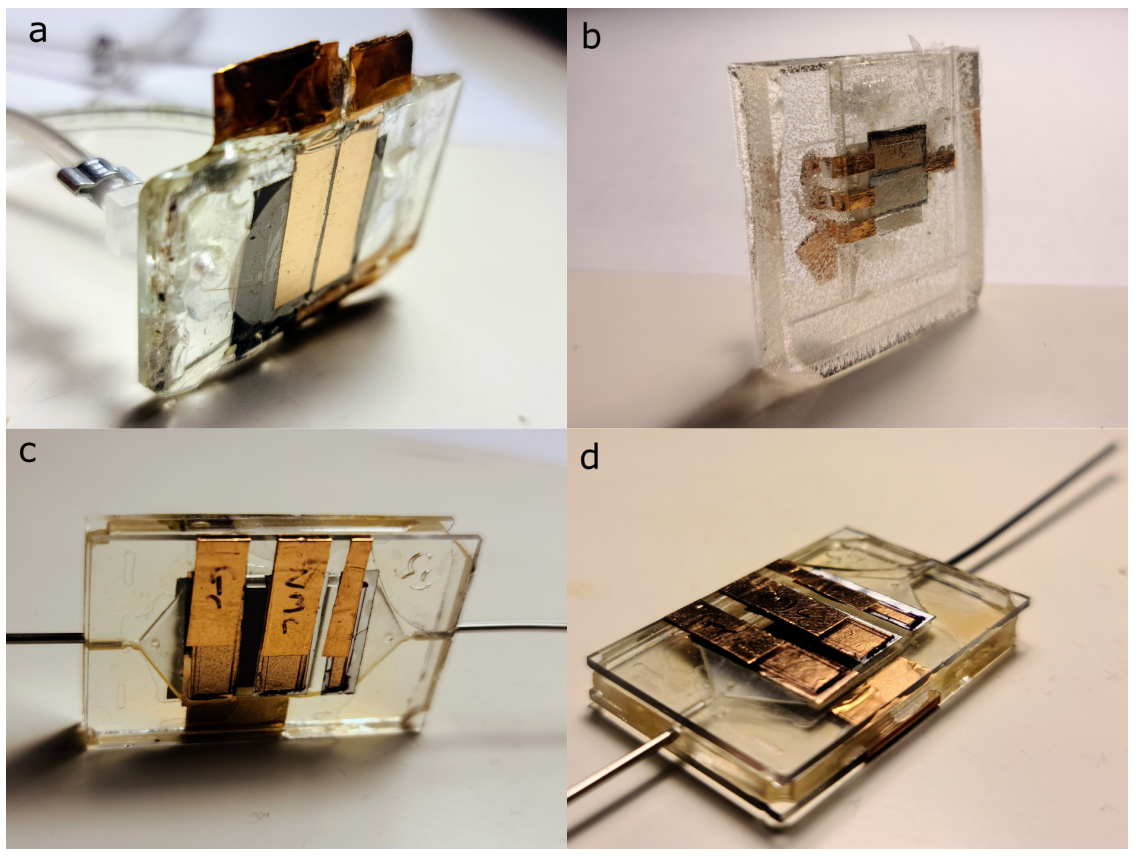

Figure 2: Photographs of partially- and fully-assembled flow cell prototypes. Copper tape leads allow potentiostatic control of flow cell working electrodes. The plastic tubing melts below the $100^{\circ} \mathrm{C}$ PDMS curing temperature, and is thus added after curing. (a) Bottom of partially assembled flow cell prior to PDMS encapsulation; (b) PDMS encapsulated flow cell; (c) Partially assembled flow cell using 3D-printed flow channel showing two working and one reference electrode; (d) Assembled and sealed flow cell with 3D-printed flow channel.

approach. The glass slides were adhered to the main flow channel by applying the same photopolymer resin used to print the channel and then the cell was cured and sealed under UV lamp. Performance of this new approach will be reported in upcoming work.

Once a flow cell was assembled, tubing was connected to the inlet and outlet fittings and the entire assembly was transported into an Ar-filled glovebox. Although none of the flow cell parts in this prototype are airreactive, most liquid electrolytes used in Li-ion batteries can absorb moisture which negatively impacts battery performance, so all electrochemical testing was performed in an inert glovebox environment. 


\section{$2.3 \quad$ Electrochemical measurements}

The flow cells were initially filled with LP 30 electrolyte (1.0 M LiPF6 in $50: 50$ v:v ethylene carbonate:dimethyl carbonate, Gotion) at a rate of 6 $\mu \mathrm{L} / \mathrm{min}$ via syringe pump. For each test, once electrolyte was observed exiting the outlet fitting, the pump was turned off and the potentiostat measured the open circuit potential (OCP) of both working electrodes. Once the OCP of each electrode achieved steady-state (i.e. $\frac{d V}{d t}<1 \frac{m V}{\min }$ ), the pump was restarted and testing the flow cell began.

Formation cycling was initially performed on each working electrode by cycling in a half-cell configuration at $\mathrm{C} / 10$ for 5 cycles. The geometric surface area of each working electrode is approximately $40 \mathrm{~mm}^{2}$, and based on loading data from the manufacturer, the negative graphite electrode was capacity limiting. The $1 \mathrm{C}$ rate for the flow cell was calculated for the 40 $\mathrm{mm}^{2}$ graphite negative electrode to be $1.15 \mathrm{~mA}$. After formation cycling, a 1 -hr rest was performed before cycling continued for 15 cycles at a rate of C/3 for a total of 20 cycles.

\subsection{Computational details}

COMSOL Multiphysics software was used to simulate various flow cell configurations and investigate how design parameters impacted device performance. The geometric collection efficiency, the total expected current at each electrode, and the concentration and velocity profiles were simulated in COMSOL for a range of design parameters including entrance length, flow channel height and width, and electrolyte flow rate.

\section{Results and discussion}

\subsection{Design considerations and calculations}

COMSOL software was used to predict device performance based on geometry and flow rate. The simulation domain was a cross-section of the flow channel spanning the length of the cell from inlet to outlet, including two coplanar working electrodes and a counter electrode opposite to the working electrodes, as shown in Figure 3. The main geometric parameters of interest were the inter-electrode spacing, $d_{g a p}$, the electrode length, $l_{\text {elec }}$, and the height of the flow channel, $h_{\text {chan }}$, since they directly affect the collection efficiency, $N_{C}$, and surface-area to volume ratio, $N_{S A V}$. For all simulations, electrolyte parameters were sourced from manufacturer specifications, dilute 
species diffusivities were calculated from experiment, and electrochemical reactions were assumed to be isothermal with temperature set at $298.15 \mathrm{~K}$.

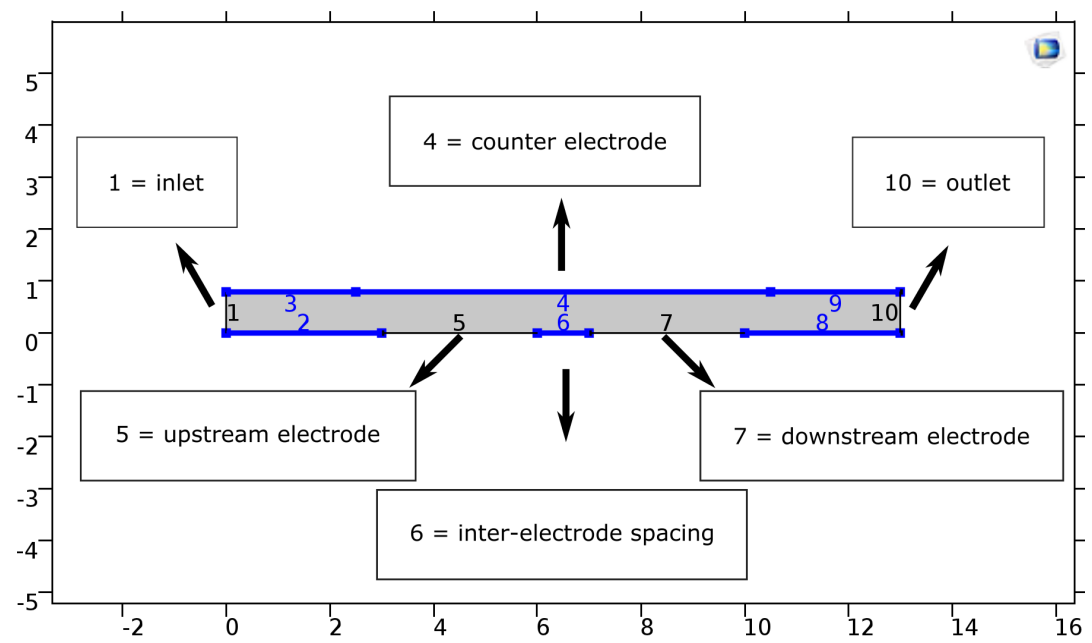

Figure 3: COMSOL simulation domain representing cross-section of flow cell. Electrodes, inlet, and outlet regions are indicated by arrows and numbering.

\subsubsection{Effect of flow rate on predicted collection efficiency}

The COMSOL model was used to investigate the effect of flow rate on collection efficiency. In this study, the geometry of the cell was fixed with the following parameters: $d_{\text {gap }}=1 \mathrm{~mm}, l_{\text {elec }}=3 \mathrm{~mm}$, and $h_{\text {chan }}=0.791 \mathrm{~mm}$. Preliminary testing found that a $1 \mathrm{~mm}$ inter-electrode spacing was reasonably large for manual electrode placement and manipulation, while small enough for product detection at the downstream electrode. Additionally, this electrode length was chosen due to practical considerations of material handling, while the channel height is $1 / 32$ ", a standard gasket size, to simplify device manufacturing.

To calculate the device's theoretical collection efficiency, a simulation was built where dilute ferrocene (FCN) is oxidized to ferrocenium (FCM) at the upstream electrode (generator) and then the generated ferrocenium is reduced at the downstream electrode (collector). This is accomplished by introducing a known concentration of the ferrocene molecule, $C_{F C N, \infty}$, in the inlet stream and instituting flux and concentration boundary conditions at the electrodes as shown in Table 1. Comparison of the oxidation and reduction currents at each electrode leads to calculation of a collection 
efficiency according to:

$$
N_{C}=\frac{-i_{\text {collector }}}{i_{\text {generator }}}
$$

Table 1: Concentration and flux boundary conditions instituted to predict collection efficiencies for the flow cell. Boundary locations are from Figure 3

\begin{tabular}{c|c|c} 
Boundary & Condition & Value \\
\hline 1 & Conc. & $C_{F C N}=C_{F C N, \infty}$ \\
5 & Conc. & $C_{F C N}=0$ \\
5 & Flux & $J_{F C M}=-J_{F C N}$ \\
7 & Conc. & $C_{F C M}=0$ \\
7 & Flux & $J_{F C N}=-J_{F C M}$ \\
Domain & Conc. & $C_{F C N}+C_{F C M}=C_{F C N, \infty}$
\end{tabular}

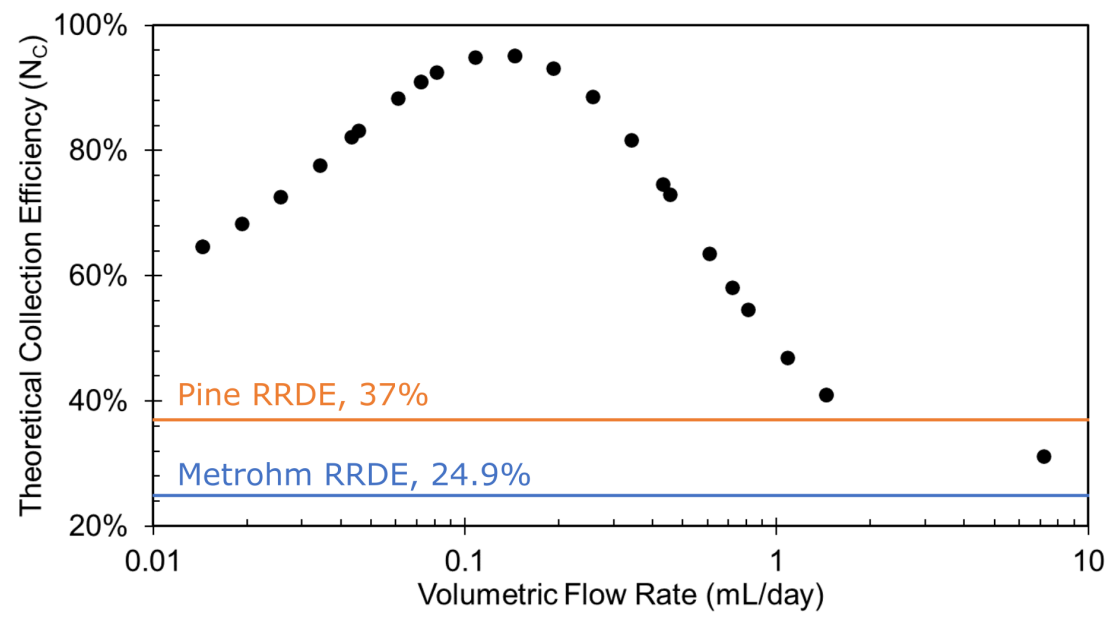

Figure 4: Predicted theoretical collection efficiencies in the flow cell device via COMSOL simulation for various flow rates. Collection efficiencies for two commercial RRDE systems are shown in orange (Pine) and blue (Metrohm).

FCN oxidation and FCM reduction reactions at the upstream and downstream electrodes were simulated over a range of flow rates from $4 \mu \mathrm{L} / \mathrm{hr}$ (i.e., the minimum flow rate of the syringe pump) to $500 \mu \mathrm{L} / \mathrm{hr}$. Since flow experiments using the flow cell mimic battery cycling, which can take weeks to complete, it can be more intuitive to represent the flow rates on a daily 
volume basis, such as $\mathrm{mL} /$ day. The COMSOL simulation of the FCN and FCM redox reactions predicted a maximum theoretical collection efficiency for a flow rate of approximately $0.1 \mathrm{~mL} /$ day, as shown in 4 . Additionally, these simulations show that collection efficiencies greater than $40 \%$ are possible for this geometry at even moderate flow rates. While the existence of an optimal flow rate for collection at the downstream electrode may seem surprising, the concentration profiles of FCM in Figure 5 visually represent how flow rate impacts collection efficiency, and help explain why an optimum exists.

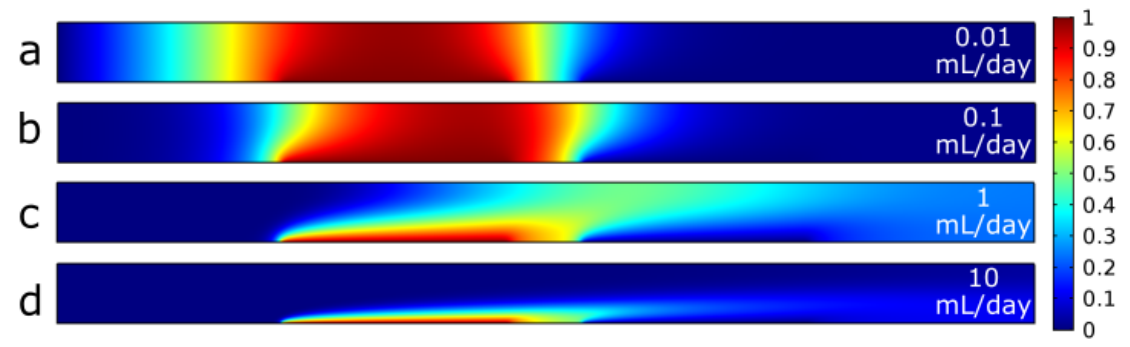

Figure 5: Predicted FCM concentration profiles in the flow cell device via COMSOL simulation for various flow rates. (a) $0.01 \mathrm{~mL} /$ day; (b) $0.1 \mathrm{~mL} /$ day; (c) 1 $\mathrm{mL} /$ day; (d) $10 \mathrm{~mL} /$ day. Concentration units are dimensionless, with the maximum concentration of 1 indicating equivalence with the bulk concentration of FCN in the inlet stream $\left(C_{F C N, \infty}\right)$.

In Figure 5, the concentration of FCM is mapped across the crosssectional area that defines the simulation domain. According to Figure 4, the simulation in Figure 5b predicted a collection efficiency of over $90 \%$ while the simulation in Figure 5c predicted a collection efficiency under $50 \%$. The concentration profiles complement those predictions by demonstrating that more FCM exits the device unreacted at a flow rate of $1 \mathrm{~mL} /$ day compared to $0.1 \mathrm{~mL} /$ day. Figure 5d also demonstrates that at high flow rates, a considerable quantity of FCM generated at the upstream electrode is convected downstream to the outlet without being reduced at the downstream electrode. Additionally, both Figures $5 \mathrm{a}$ and 5b show back-diffusion of FCM toward the inlet (cyan, green, and yellow to the left of dark red region) at low flow rates, indicating that a flow rate of $1 \mathrm{~mL} /$ day is sufficient to prevent back-diffusion of reacted species. These predictions from COMSOL simulations were important tools for designing the prototype flow cell device and understanding how operational parameters like flow rate affect device performance. 


\subsubsection{Surface-area to volume ratio optimization}

The primary advantages of this novel device, over traditional electroanalytic tools, are the high collection efficiencies $\left(N_{C}\right)$ that can be obtained, as shown above, and a more realistic surface-area-to-volume ratio, $N_{S A V}$. In practical batteries such as coin cells, the $N_{S A V}$ is on the order of 10,000 $\mathrm{cm}^{2} / \mathrm{cm}^{3}$, while a typical $10 \mathrm{~mL}$ RRDE experiment is on the order of 0.01 $\mathrm{cm}^{2} / \mathrm{cm}^{3}$. This 6 order of magnitude difference represents one of the major experimental drawbacks of using RRDE techniques to study battery interfaces. Therefore, fabricating a flow cell device with an $N_{S A V}$ ratio on the order of $10^{1}-10^{2} \mathrm{~cm}^{2} / \mathrm{cm}^{3}$ would be a remarkable improvement in reactor design for electroanalysis of battery reactions.

Thus, the flow cell prototype presented here was designed to dramatically increase the $N_{S A V}$ over a typical RRDE and better approximate the environment of a practical battery. For simplicity, both working electrodes were modeled in COMSOL as planar, unpassivated electrodes, therefore $N_{S A V}$ was calculated according to:

$$
N_{S A V}=\frac{w_{\text {elec }} l_{\text {elec }}}{w_{\text {elec }} l_{\text {elec }} h_{\text {chan }}}
$$

where $w_{\text {elec }}$ is the width of the electrode (perpendicular to direction of flow), $l_{\text {elec }}$ is the length of the electrode (parallel to direction of flow), and $h_{\text {chan }}$ is the height of the channel. Clearly, the $N_{S A V}$ scales inversely with the channel height, and thus decreasing the height of the flow channel will increase this critical ratio to more practical levels. According to Equation 1, an $N_{S A V}$ ratio on the order of $10^{1}-10^{2} \mathrm{~cm}^{-1}$ would be achieved by constructing devices with channel heights ranging from $10^{-1}$ to $10^{0} \mathrm{~mm}$. For the system modeled here, the $h_{\text {chan }}$ was set at $1 / 32$ ", or $0.791 \mathrm{~mm}$, which satisfies the conditions for vastly improving the $N_{S A V}$ ratio from that of a typical RRDE. Therefore, the flow cell prototype presented here not only can access very high collection efficiencies at even moderate flow rates, but also improves upon the $N_{S A V}$ ratio of the RRDE by up to 4 orders of magnitude.

\subsection{Device calibration}

Prior to cycling the flow cell in an operational mode similar to a LIB, the system was calibrated using the well understood single electron redox reaction of decamethylferrocene (dmFCN) to decamethylferrocenium (dmFCM).[7-9] The purpose of calibrating the device was to ensure communication between the two electrodes followed theoretical predictions. Therefore, an electrolyte 
solution containing $1 \mathrm{mM}$ dmFCN was pumped through the cell at a flow rate of $4 \mu \mathrm{L}$ per min. In the calibration test, the upstream graphite electrode was cycled around its equilibrium redox potential, approximately $2.8 \mathrm{~V}$ vs. $\mathrm{Li} / \mathrm{Li}^{+}$. Due to Li's high reactivity with air and moisture, a semi-delithiated $\mathrm{Li}_{0.5} \mathrm{FePO}_{4}$ electrode is used as a pseudo-reference electrode, which we have measured to have an equilibrium potential of $3.45 \mathrm{~V}$ vs. $\mathrm{Li} / \mathrm{Li}^{+}$. Therefore, the upstream electrode was cycled between -0.8 and -0.5 vs. $\mathrm{Li}_{0.5} \mathrm{FePO}_{4}$, corresponding to 2.65 and $2.95 \mathrm{~V}$ vs. $\mathrm{Li} / \mathrm{Li}^{+}$. The commercial electrolyte, LP 30, is non-reactive in this potential window (not shown), thus the current observed at the upstream graphite electrode is attributed to $\mathrm{dmFCN}$ oxidation and dmFCM reduction.

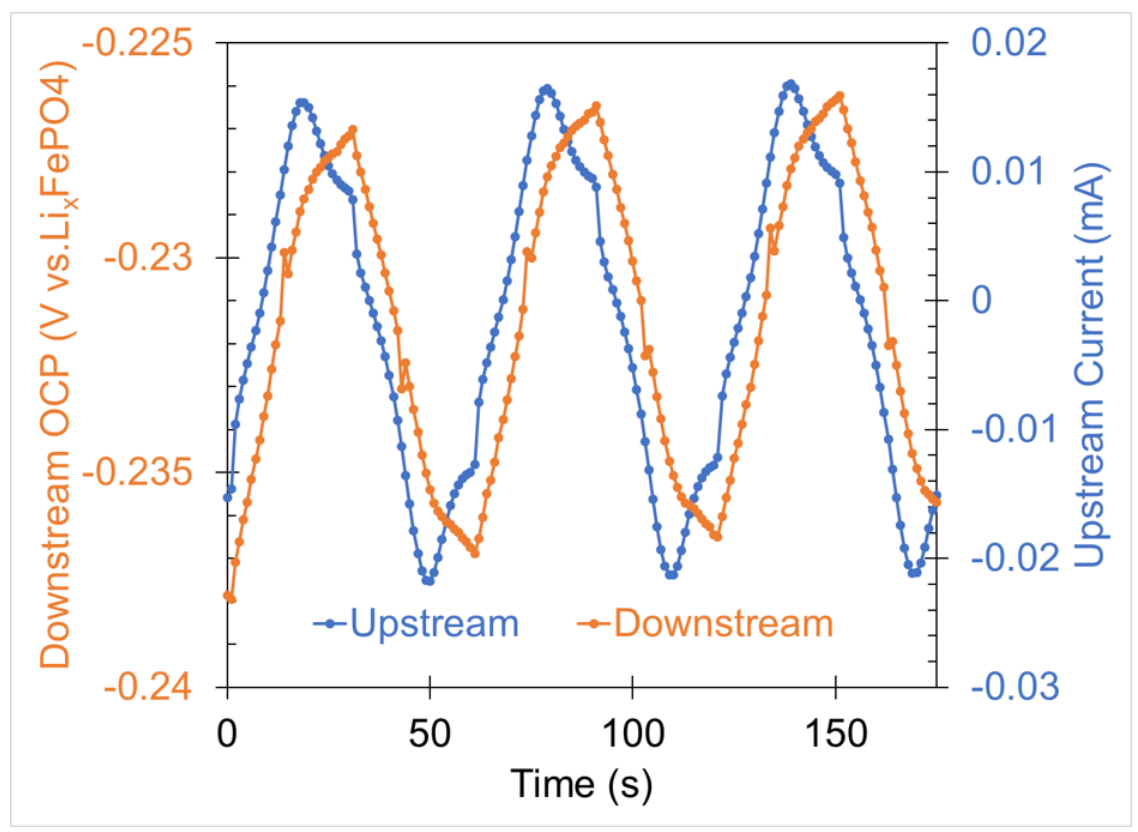

Figure 6: Open circuit potential of the downstream NMC electrode (orange) responds to dmFCN redox activity at upstream graphite electrode (blue).

While the upstream graphite electrode is performing cyclic voltammetry, the downstream NMC electrode is resting and the open circuit potential (OCP) of the electrode is measured. The current measured at the upstream electrode and the downstream electrode's OCP are shown in Figure 6 in blue and orange, respectively. When the upstream electrode measures a positive current, the downstream electrode has a corresponding increase in open circuit potential. When the cyclic voltammogram direction flips 
at the vertex potential, the current decreases and then becomes negative. The downstream electrode records a corresponding decrease in OCP. These phenomena are not synchronous but rather occur with the same periodicity and are offset temporally by a fixed amount.

In fact, the peak-to-peak separation (orange-to-blue) in Figure 6 can be related to device design parameters. Specifically, the peak-to-peak separation is a time constant for convection between electrodes and is a function of flow rate, inter-electrode distance, channel width, and channel height according to:

$$
\tau=\frac{1}{\delta} \frac{\dot{V}}{w_{\text {chan }} h_{\text {chan }}}
$$

where $\tau$ is the convective time constant, $\delta$ is the inter-electrode distance (1

$\mathrm{mm}), \dot{\mathrm{V}}$ is the volumetric flow rate $(4 \mu \mathrm{L} / \mathrm{min}), w_{\text {chan }}$ is the channel width $(15.4 \mathrm{~mm})$, and $h_{\text {chan }}$ is the channel height $(0.050 \mathrm{~mm})$. Under these conditions, the calculated time constant is 11.6 seconds, while the average peakto-peak separation measured in Figure 6 was 12 seconds. This calibration is preliminary evidence that the prototype can perform generator-collector electrochemical experiments. Next, we apply these capabilities to studying LIB reactions.

\subsection{Battery cycling mode}

One of the main applications of the electrochemical flow cell developed here is to perform battery cycling protocols on commercial battery electrodes under varying flow conditions and geometries. The flow cell enables the investigation of battery reactions with controlled inter-electrode communication, something that is not possible with tradition battery testing formats. This can be a powerful tool for diagnosing crosstalk reactions and to gain further understanding into the mechanisms that promote parasitic side reactions.

In this study, we compare the cycling performance of the graphite negative electrode in two configurations: upstream to the NMC electrode and downstream from the NMC electrode. Figure 7 shows initial charge-discharge cycling data for the graphite electrode in upstream configuration. The first cycle charge and discharge capacities are both greater than theoretical (1.15 $\mathrm{mAh}$ ), which is evidence of SEI formation and significant side reactions. Under flow conditions an increase in current attributed to side reactions is expected. In a stagnant battery, side reactions may be thermodynamically limited by local concentration gradients and reactant or product solubilities, yet under forced convection these limitations may be removed. While the $N_{S A V}$ ratios of device prototypes presented here are still a few orders 


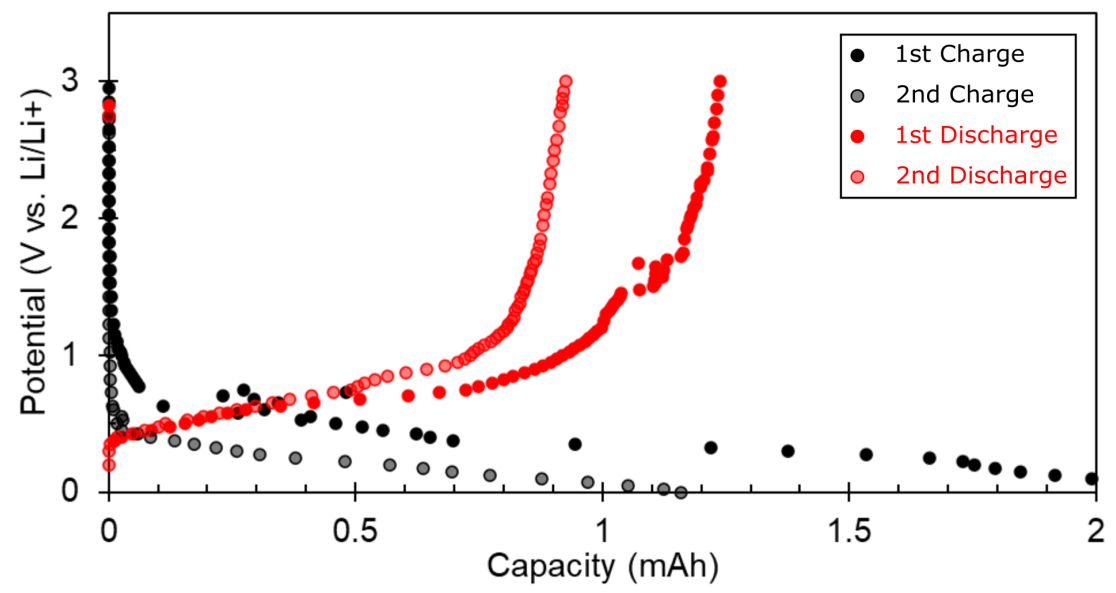

Figure 7: Initial charge-discharge cycling of graphite electrode in flow cell. Graphite is upstream to NMC electrode.

of magnitude lower than practical coin cells, they still represent a major improvement over a typical RRDE.

After SEI formation, the graphite electrode continues to perform charge and discharge cycles, however at $\mathrm{C} / 3$ rate. While the graphite electrode is charging and discharging, the NMC electrode is set to a fixed potential of $1.45 \mathrm{~V}$ vs. $\mathrm{Li}_{0.5} \mathrm{FePO}_{4}$, or approximately $4.9 \mathrm{~V}$ vs. $\mathrm{Li} / \mathrm{Li}^{+}$. This high voltage was selected to maximize the effects of metal dissolution from the NMC electrode. In another flow cell prototype, graphite was cycled downstream from the NMC electrode so that crosstalk from NMC to graphite may be observed. Figure 8 shows the 17 post-formation cycles performed by a graphite electrode downstream from an NMC electrode undergoing high-voltage oxidation. Capacity fade with each cycle is apparent, and the final discharge capacity (cycle 20) is half that of the first post-formation discharge capacity (cycle 4).

The effects of electrode orientation can be clearly seen when comparing the capacity retention of the graphite electrode under the two geometries, as in Figure 9. Here the last SEI formation cycle (3) is considered cycle 0, thus cycle 4 is the 1st post-formation cycle. When the graphite negative electrode is situated downstream from the NMC electrode (i.e. positive-to-negative communication is allowed) capacity fade from cycle to cycle is easily observed and comports with expectations based on reports in the literature and prior work. [3] When the graphite negative electrode is situated upstream from the NMC electrode (i.e. positive-to-negative communication is blocked), there 


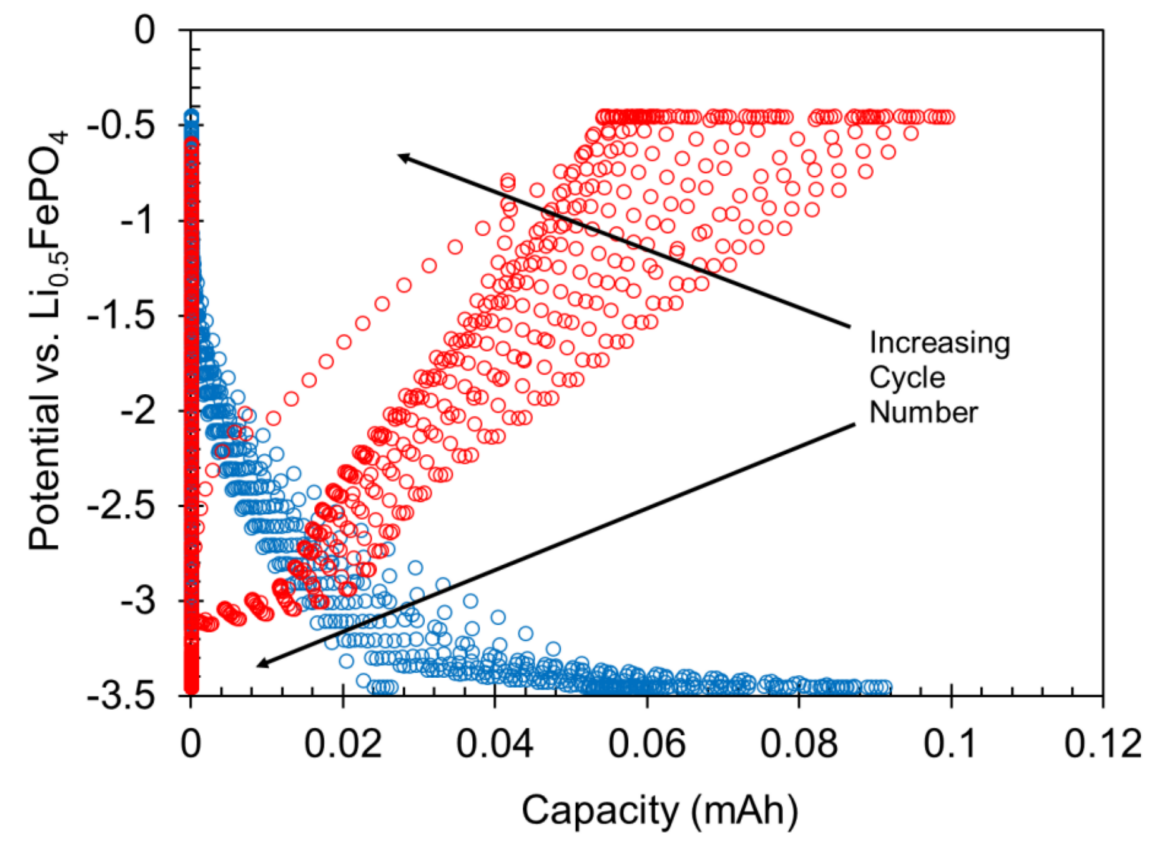

Figure 8: Post-formation cycling (cycles 4-20) of a graphite electrode downstream from NMC electrode shows severe capacity fading. NMC electrode undergoes highvoltage oxidation to promote metal dissolution.

is some fading in the first several post-formation cycles, however by postformation cycle 10 the capacity has already recovered. This is clear evidence that the electrochemical flow cell presented here is capable of replicating a battery environment and can detect pernicious crosstalk reactions such as metal dissolution and deposition.

\subsection{Limitations and path forward}

While the prototype presented here is able to detect capacity fade induced by the metal dissolution crosstalk mechanism, there are various limitations currently impeding its utility and broader application. For instance, continually increasing the $N_{S A V}$ ratio of the device through careful engineering and fabrication will help ensure measurements are collected in environments most similar to true battery use. Once cell fabrication is optimized for this four-electrode approach, testing can begin to determine specific kinetic rate constants and transport coefficients for crosstalk reactions and species, respectively. This data will be vital to the increasing efforts at developing 


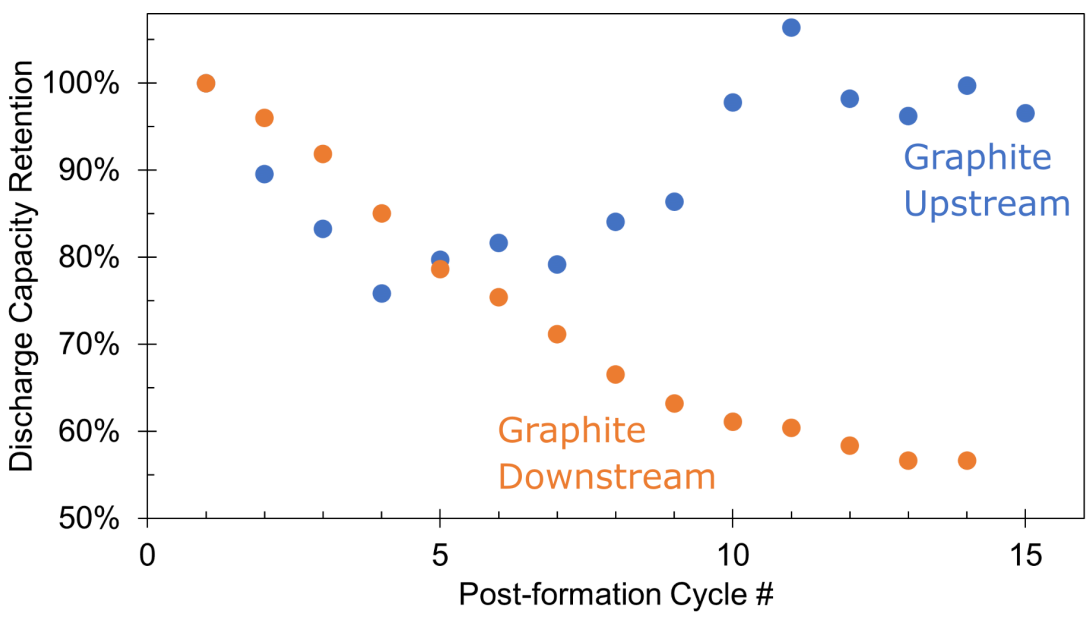

Figure 9: Influence of graphite position on capacity retention when cycling. Discharge capacity of graphite electrode upstream to NMC (blue) recovers, while the discharge capacity of graphite downstream from NMC (orange) fades continually.

predictive physics-based models for LIB lifetime prediction. The capabilities of the flow cell to detect reactions and products can also be enhanced by adding collector electrodes and gas ports, which can perform electrochemical product detection between cycling electrodes or be fed to chromatography and spectroscopy instruments, respectively. Ultimately, the future of flow reactors for characterizing crosstalk reactions in Li-ion batteries lies in engineering reactor environments that mimic stagnant Li-ion batteries while increasing device functionality for product detection.

\section{Conclusions}

Improvements to Li-ion battery lifetime have reached a seeming upper-limit due to experimental limitations of the battery itself. Here we presented the development of an electrochemical flow cell for studying complicated battery reactions that are untenable to interrogate using traditional battery testing methods. The flow cell is comprised of two working electrodes made from commercial battery electrode sheets and a dedicated pseudo-reference electrode. The design of the flow cell was assisted by finite element simulations of the flow field over the two working electrodes for various geometries. Assembled flow cells were calibrated using a reversible decamethylferrocene redox reaction between the two working electrodes. Finally, the flow cell was 
cycled like a battery to probe the effect of inter-electrode communication on capacity fading of a graphite negative electrode and we showed that the flow cell prototype can replicate and detect crosstalk in advanced Li-ion battery systems. Future improvements to flow cell design will optimize the $N_{S A V}$ while testing will measure important kinetic rate constants and transport coefficients of crosstalk reactions and species. 


\section{References}

(1) Harris, O. C.; Lee, S. E.; Lees, C.; Tang, M. Journal of Physics: Energy 2020, 2, 032002.

(2) Dumitrescu, I.; Yancey, D. F.; Crooks, R. M. Lab on a Chip 2012, 12, 986.

(3) Harris, O. C.; Tang, M. H. The Journal of Physical Chemistry C 2018, 122, 20632-20641.

(4) Lu, P.; Li, C.; Schneider, E. W.; Harris, S. J. Journal of Physical Chemistry $C$ 2014, 118, 896-903.

(5) Wang, L.-F.; Ou, C.-C.; Striebel, K. A.; Chen, J.-S. Journal of The Electrochemical Society 2003, 150, A905.

(6) Wandt, J.; Freiberg, A.; Thomas, R.; Gorlin, Y.; Siebel, A.; Jung, R.; Gasteiger, H. A.; Tromp, M. J. Mater. Chem. A 2016, 4, 18300-18305.

(7) Noviandri, I.; Brown, K. N.; Fleming, D. S.; Gulyas, P. T.; Lay, P. A.; Masters, A. F.; Phillips, L. Journal of Physical Chemistry B 1999, 103, 6713-6722.

(8) Bond, A. M.; Marken, F. Journal of Electroanalytical Chemistry 1994, 372, 125-135.

(9) Inkpen, M. S.; Albrecht, T.; Long, N. J. Organometallics 2013, 32, 6053-6060. 